http://dx.doi.org/10.18778/2196-8403.2007.07

MONIKA SZCZEPANIAK

\title{
Ulanen und Stahlhelden. Konstruktion der polni- schen und deutschen militärischen Männlichkeit im Kontext des Ersten Weltkrieges
}

\begin{abstract}
Artykuł stanowi pierwszą próbę rozpoznania podobieństw i różnic w konstytuowaniu się polskiej i niemieckiej męskości militarnej w szeroko pojętym kontekście kulturowym pierwszej wojny światowej. Podstawowe pytanie dotyczy kwestii ogólnej militaryzacji męskości i konstrukcji ,twardego ' maskulinizmu, który w niemieckich warunkach kryzysu męskości był dominujący, a w polskich warunkach nadziei ,przed złotym czasem“ (STANISŁAW DŁUGOSZ 1917) nie odegrał znaczącej roli. Teza o hegemonialnych paradygmatach polskiego porywczego ułana i niemieckiego „stalowego bohatera” dyskutowana jest na przykładach tekstów literackich ideologizujących każdorazowo „pożądaną" męskość bohatera walki ze wskazaniem na próby dekonstrukcji tych paradygmatów.
\end{abstract}

Der Beitrag unternimmt einen ersten Versuch, Ähnlichkeiten und Unterschiede in der Konstituierung der deutschen und polnischen militärischen Männlichkeit im breit gefassten kulturellen Kontext des Ersten Weltkrieges zu erkennen. Das Grundanliegen bezieht sich auf das Problem der allgemeinen Militarisierung von Männlichkeit und die Konstruktion eines ,harten‘ Maskulinismus, der unter den Bedingungen der Männlichkeitskrise in Deutschland dominierte und im polnischen Raum, wo man auf die so genannte ,goldene Zeit“ (StanisŁaw DŁugosz 1917) hoffte, wohl keine bedeutende Rolle spielte. Die hegemonialen Paradigmen des polnischen verwegenen Ulanen einerseits und des deutschen Stahlhelden andererseits werden am Beispiel von literarischen Texten diskutiert, die die jeweils erwünschte Männlichkeitsvariante des kämpfenden Helden ideologisieren. Hingewiesen wird aber auch auf literarische Versuche, diese Paradigmen zu dekonstruieren.

The article is the first attempt of recognition of similarities and differences in forming Polish and German military masculinity, in the widely understood culture context of WWI. The fundamental question here refers to the issue of general militarization of 
Monika Szczepaniak

masculinity and the constructing of , hard ' masculinity ideology, which was dominant in the atmosphere of masculinity crisis in Germany and, in Poland, in the atmosphere of hope „before the golden time“" (STANiSŁAW DŁugosz 1917) did not play the crucial role. The thesis on hegemonic paradigms of a Polish impetuous lancer and a German „,steel hero" is discussed on the basis of literary texts, which always offer ideologization of the „,desired“ masculinity of a battle's hero, pointing at the attempts of deconstructing these paradigms.

Auf den ersten Blick mag die im Titel formulierte These von verschiedenen militärischen Männlichkeiten, die sich im gleichen historischen Kontext im Rahmen verschiedener nationaler Diskurse entwickeln, befremdend wirken. Der Mann als ,eisernes Geschlecht', als Ritter, Krieger und Soldat scheint ein Ideal zu sein, das schon immer das männliche Handlungsrepertoire in Europa nachhaltig beeinflusste und den männlichen Habitus mitkonstituierte. ${ }^{1}$ In der Sprache des australischen Soziologen Robert W. Connell handelt es sich um hegemoniale Männlichkeit, die sich nicht unbedingt durch direkte Anwendung von Gewalt gegenüber Frauen oder schwächeren Geschlechtsgenossen realisiert, sondern durch eine Reihe von systeminternen Mechanismen unterstützt wird und nicht zuletzt auf männlicher Autorität aufbaut, die ein großes Maß an Zustimmung der Beherrschten voraussetzt. CoNNELL (1999:91) schlägt vor, Männlichkeit und Weiblichkeit als Geschlechterprojekte aufzufassen:

Statt zu versuchen, Männlichkeit als ein Objekt zu definieren (ein natürlicher Charakterzug, ein Verhaltensdurchschnitt, eine Norm), sollten wir unsere Aufmerksamkeit auf die Prozesse und Beziehungen richten, die Männer und Frauen ein vergeschlechtlichtes Leben führen lassen. ,Männlichkeit' ist - soweit man diesen Begriff in Kürze überhaupt definieren kann - eine Position im Geschlechterverhältnis; die Praktiken, durch die Männer und Frauen diese Position einnehmen, und die Auswirkungen dieser Praktiken auf die körperliche Erfahrung, auf Persönlichkeit und Kultur.

Folgerichtig ist die ,hegemoniale Männlichkeit ' als der in der jeweiligen Kultur dominierende Männlichkeitstypus zu verstehen (z.B. heterosexuell und weiß; ritterlich und soldatisch etc.), dessen Konstruktion und Popularisierung die bestehende Geschlechterhierarchie absichert und der nicht unbedingt mit individuell realisierten Lebensentwürfen übereinstimmt. Mit den Worten von CONNELL (1999:98): „Hegemoniale Männlichkeit kann man als jene Konfiguration geschlechtsbezogener Praxis definieren, welche die momentan akzep-

1 Eine der wichtigsten Manifestationen dieses Paradigmas ist die gesamteuropäische Duellpraxis, die auf dem Verhaltenskomplex "Ehre und Wehrhaftigkeit" gründete und Gewalttätigkeit mit einem detailliert ausgearbeiteten Kodex von Regeln verband. Vgl. dazu FrEVERT (1991). 
tierte Antwort auf das Legitimitätsproblem des Patriarchats verkörpert und die Dominanz der Männer sowie die Unterordnung der Frauen gewährleistet (oder gewährleisten soll).“ Die These von einer militärischen Männlichkeit im Umfeld des Ersten Weltkrieges lässt sich jedoch diskutieren. Die Geschichte der polnischen Männlichkeit wurde bisher kaum untersucht, und es liegen noch keine wissenschaftlichen Publikationen über die historischen Männlichkeitskonstruktionen in Polen vor. Die historischen Männlichkeiten in der polnischen Kultur und Literatur, d.h. auf der Ebene der Repräsentation zu untersuchen, ist daher mit beträchtlichen Schwierigkeiten verbunden. Dennoch gibt es guten Grund zu behaupten, dass sich das Verhältnis von Militär, Politik und Geschlechtersystem in Polen und Preußen-Deutschland schon seit dem 19. Jahrhundert unterschiedlich entwickelte. Seit der allgemeinen Einführung des Wehrdienstes in Deutschland wird der männliche Geschlechtscharakter zunehmend „militarisiert“ und das Referenzsystem Staat - Nation - Politik (HAGEMANN 1996:51-68) in den Status einer für den männlichen Lebenszusammenhang besonders relevanten Konstellation erhoben, die die Männer an das Staatswesen koppelt, der Verweiblichung entgegenwirkt und Männergemeinschaften fördert. ERNST HANISCH (2005:19) konstatiert: „Der Entwurf des Staates zielte auf den Zusammenhalt der wehrfähigen Männer. [...] Wehrpflicht und Nationsbildung liefen in Preußen parallel. Die Armee wurde zur ,Schule der Nation" hochstilisiert.“

In der entsprechenden historischen Periode kann in Polen vom Referenzsystem Staat - Nation - Politik keine Rede sein. Die polnische Männlichkeit konstituiert und entwickelt sich ohne Staat, sie kann aber auf eine nationale Tradition zurückblicken und ist in den aktuellen nationalen Diskurs verwickelt, der natürlich die Unabhängigkeit Polens als Staat stets anvisiert. Ein Diskurs, der den Staat als Sehnsuchtsfigur charakterisiert, bringt andere Männlichkeitskonzepte hervor als ein Diskurs, in dem der Staat als disziplinierende, vermännlichende Instanz begriffen wird, die nach Gehorsam und Opfer verlangt. Vielleicht könnte dieser Unterschied folgendermaßen formuliert werden: Der preußische ,militärische“ Geist fördert eiserne Helden, Männer aus Stahl, ,Kältemaschinen“ (LETHEN 1990); die polnische romantische Tradition des Patriotismus eines unterdrückten Volkes bringt verträumte Aufständische, „wahnsinnige Patrioten“ (JANION 1991:189), todgeweihte Don Quijotes hervor. Während der Erste Weltkrieg in Deutschland als ein Ereignis stilisiert wird, das die Männer „endlich stählern eint“ (DEHMEL 1914, zit. nach KrocKOw 1995:16), folgen 
die polnischen „bildhübschen Jungs“ dem „Krieglein““2 als einer umwerfend schönen Frau. ${ }^{3}$ In beiden Fällen haben wie es mit einer spezifischen Ästhetisierung des Männerbildes zu tun. Dies möchte ich zunächst als Interpretationsthese gelten lassen, die sich jedoch im vorliegenden Beitrag nicht endgültig verifizieren lässt. Vielmehr wird versucht, an einigen Beispielen zu zeigen, welche Komponenten aus dem kulturellen Katalog von ,männlichen ‘ Attributen zum einen die polnische und zum anderen die deutsche Männlichkeit ausmachen und inwiefern in beiden nationalen Kontexten von harter, kriegerischer Maskulinität die Rede sein kann.

\section{„Überwinder, Stahlnaturen“ (JÜNGER 1922:32)}

Im wilhelminischen Deutschland wird ein heroisches Männerbild verbreitet, das in anderen Nationen in dieser Form kaum auffindbar ist. Der Krieger erscheint als männlicher Lebensberuf (bzw. Berufung) schlechthin, das Soldatendasein erfährt eine spezifische und neuartige Ästhetisierung unter Berücksichtigung verschiedener Aspekte des "technoromantischen Abenteuers“ (KRAUS 1918). Der Krieg wird ideologisch als Kampf gegen die körperliche und moralische Degeneration stilisiert, als ein Ereignis, das der zunehmenden Schwächung und Effeminierung des männlichen Geschlechts ein Ende setzen soll. Diese Hoffnung auf die therapeutische Funktion des Krieges hat weitreichende Implikationen: Man will sich zum Beispiel befreien vom weiblich konnotierten emotionalen Treibhausklima der bürgerlichen Familie, um auf dem Schlachtfeld den ,élan vital ' des Mannes zu stärken. Der Große Krieg wird (auch nachträglich) als ein intensives Erlebnis, ein großes Abenteuer, eine besondere Art Prüfstein für Männlichkeit ideologisiert. Die entsprechend sozialisierte deutsche „eiserne Jugend“4 wird mit chauvinistischen Reden in den

2 Vgl. das berühmte Kriegslied Wojenko, wojenko, cóżeś ty za pani (Krieglein, Krieglein, was bist du für eine Dame), das fast jeder Pole kennt und das nach wie vor oft gesungen wird.

3 In Deutschland wird heftig gegen die Vision der Verweiblichung der Kultur gekämpft, um die bedrohte Männlichkeit zu verteidigen. Nach dem Krieg wird die Weimarer Republik als weiblich dargestellt (Frau Republik) und die regressive Rückkehr zur Großen Mutter beklagt. Das Bedürfnis nach Re-Virilisierung der Kultur manifestiert sich nicht zuletzt im Comeback der Kriegsthematik und der erneuten Idealisierung des Soldaten.

4 Das Herausbilden des Untertanen-Typus im wilhelminischen Deutschland schildert der Roman Der Untertan (1914) von Heinrich Mann, in dem die institutionelle Rolle solcher Instanzen wie Schule, Universität, Korporation und Militär ge- 
„großen Krieg der weißen Männer“5 geschickt. „Der technische Krieg wurde mit Gas und Torpedos geführt, aber die Phrase zog das Schwert" (HANISCH 2005:29) und überhöhte den einzelnen Kämpfer unter Rekurs auf heroische Traditionen der Kriegsdichtung. WILHELM WORRINGER (1914/15:20) bemerkt die geschlechterpolitische Komponente des Krieges und befürchtet ein katastrophales Szenario, nach dem das Weib (die ,femme fatale') über den Helden siegt und das Feld behauptet. Trotzdem schreibt er 1914 voller Zuversicht:

Wir werden siegen in diesem Kampf; werden siegen mit den aufs höchste angespannten Muskeln unserer geistigen Energien, werden siegen nicht zuletzt auch mit der Gewalt unserer so glänzend organisierten und disziplinierten körperlichen Energien, mit jener Körperkraft, die man brutal nennen mag, die aber anbetungswürdig ist, wenn man es mit einer verzweifelten, unberechenbaren Schaden stiftenden Hysterie zu tun hat.

Disziplinierte körperliche Energien und Hysterie werden hier an entgegengesetzten Polen situiert. Hysterie und Nervosität sind - wie Joachim Radkau nachgewiesen hat - zwei große „Übel“ der wilhelminischen Ära, denen man auf verschiedene Art und Weise entgegenzuwirken versucht. Bei RADKAU (1998:104) ist zu lesen:

In der Anfangszeit des Ersten Weltkrieges grassierte ein solcher pseudomedizinischer Bellizismus, der aus der Balneologie die ,Stahlbad'-Metapher übernahm; selbst ein renommierter Neurologe wie Albert Eulenburg pries den Krieg 1915 als ein mit ,fast allmächtiger Heilkraft ausgerüstetes Stahlbad [...] für die im Staub langer Friedensjahre und einförmiger Berufstätigkeit verdorrenden und verschmachtenden Nerven".

Eulenburgs illusionärer Glaube korrespondiert mit dem kulturellen Imperativ der männlichen Nervenstärke und Affektkontrolle.

Der militärische Männerraum erzeugt Inklusionen und Exklusionen und beweist dadurch einen hegemonialen Charakter. Er zwingt Männer zur Übernahme der Soldatenrolle oder zwingt sie in Rollen, die dem ganzen Leben eine besondere Wendung geben: Deserteure, Kriegsdienstverweigerer, Meuterer, Überläufer, Selbstverstümmler, Selbstmörder, gefolgt von Ausweichstrategien innerhalb der Soldatenrolle (,Kriegsneurotiker', ,Simulanten', ,Kriegsunfreiwillige‘, Stellungsflüchtige, Gehorsamsverweigerer, ,Feige‘ u. a.m.). (SCHMALE 2003:198)

zeigt wird. Eine besondere Rolle spielen die Männerbünde und die männliche Kameraderie, die dem Protagonisten das Gefühl emotionaler und sozialer Sicherheit vermitteln.

$5 \quad$ So der Titel eines Romanzyklus von Arnold Zweig (1927-1953). 
Monika Szczepaniak

Um aus dem gesellschaftlich akzeptierten Rahmen der wahren, d.h. soldatischen Männlichkeit nicht herauszufallen, musste man sich freiwillig melden, als tauglich eingestuft werden und sich an der Front bewähren. REULECKE (2001:94) zitiert ein Gedicht von Heinrich Zerkaulen, das den „Geist 1914“ atmet und in der Vorstellung eines juvenilen Heldentums schwelgt. Im Zentrum der Aufmerksamkeit steht die Mannwerdung durch den Krieg:

Ein nacktes Schwert wächst in die Hand hinein,

der Stunden Ernst fließt stahlhart durch mich hin.

Da steh ich stolz und hoch gereckt allein,

im Rausch, daß ich ein Mann geworden bin.

Wie auch immer die Realität jenseits des Kriegsmythos ausgesehen haben mag, nach dem Krieg herrschte allgemein das Gefühl vor, dass eine neue Art Mann aus den Schützengräben auferstanden sei: eben ,gestählt‘. Elemente der literarischen Konstruktion einer ,stahlharten ' Männlichkeit lassen sich in literarischen und publizistischen Texten nach 1918 finden, in denen man auf eine Heroisierung der Soldatengemeinschaft sowie Ästhetisierung und Erotisierung des Kriegserlebnisses stößt. Diese Männlichkeit kann natürlich nur ,in Stahlgewittern“ „geboren“ werden. Ernst Jüngers hochgelobte Kriegsbücher ,[...] heben besonders hervor, was es bedeutet, ein reifer Mann zu sein: Freude am Kampf zu haben, keine Gnade zu kennen“ (Mosse 1997:147). Jünger glorifiziert eine neue Männerrasse, die den Krieg hervorgebracht habe; es sind Männer, „wie sie bisher die Welt nie gesehen“, Männer aus Stahl, voller Energie, mit muskulösen Körpern, eindrucksvollen Gesichtern, ,versteinerten“ Augen:

Es war eine ganz neue Rasse, verkörperte Energie, mit höchster Wucht geladen. Geschmeidige, hagere, sehnige Körper, markante Gesichter, Augen in tausend Schrecken unterm Helm versteinert. Sie waren Überwinder, Stahlnaturen, eingestellt auf den Kampf in seiner gräßlichsten Form. [...] Jongleure des Todes, Meister des Sprengstoffes und der Flamme, prächtige Raubtiere, federten sie durch die Gräben. Im Augenblick der Begegnung waren sie der Inbegriff des Kampfhaftesten, was die Welt tragen konnte, schärfste Versammlung des Körpers, der Intelligenz, des Willens und der Sinne. (JÜNGER 1922:32f.)

Jüngers „Stahlgestalten“ haben nicht nur eine natürlich-erotische Qualität. Die mystisch-irrationale Überhöhung des Soldatentums enthält neben dem ,Raubtierhaften ' auch Elemente des ,Technischen“ oder der Unterwerfung unter das Diktat der modernen Kriegsführung. Angesichts der Modernisierung der Kriegswaffen kann sich der Jünger'sche ,neue Mann“ problemlos zurechtfinden: 
Es sind die Stahlgestalten, deren Adlerblick geradeaus über schwirrende Propeller die Wolken durchforscht, die in das Motorengewirr der Tanks gezwängt, die Höllenfahrt durch brüllende Trichterfelder wagen, die tagelang, sicheren Tod voraus, in umzingelten, leichenumhäuften Nestern halbverschmachtet hinter glühenden Maschinengewehren hocken. Sie sind die besten des modernen Schlachtfeldes, von rücksichtslosem Kämpfertum durchflutet, deren starkes Wollen sich in geballtem, zielbewußtem Energiestoß entlädt. (JÜNGER 1922:74)

Diese Passagen heben kognitive und affektive Dispositionen hervor, die für die „Hochkultur des Kampfes“ (JÜNGER 1922:63) besonders relevant sind. Allerdings ignoriert Jüngers Heroismusprojektion die Tatsache, dass die Anonymisierung und Vermassung des Kriegsgeschehens zum einen und die Dominanz der Technik zum anderen das heroische Individuum gleichsam zum Verschwinden bringen (THEEL 1993:99-118). Den von Jünger verherrlichten männlichen Helden müsste man sich vorstellen als von ,metallischer' Kälte bestimmt, ausgestattet mit einem Körper in der Funktion eines Leistungsorgans, immun gegen Gefühlsregungen, präpariert für das Walzwerk der Front, ohne Triebe, ohne Psyche, reduziert auf den ,stählernen' Leib. THEWELEIT (1980:185) meint in seiner bahnbrechenden Studie, Jünger wolle darauf hinaus, eine Utopie der Körpermaschine zu gestalten. Der mühsam konstruierte Körperpanzer wird der notwendigen Arbeit der ,Stahlnaturen“ gerecht: ,,[...] alles zu verfolgen, einzudämmen, zu unterwerfen, was sie zurückverwandeln könnte in das schrecklich desorganisierte Gewimmel aus Fleisch, Haaren, Haut, Knochen, Därmen, Gefühlen, das Mensch heißt, alter Mensch“" (THEwELEIT 1980:186). Jüngers Virilitätskonzept verdankt sich dem Impuls der schon erwähnten, sozialhistorisch und geschlechtergeschichtlich begründbaren Infragestellung männlicher Macht.

Die Kriegspropaganda konzentrierte sich auf die Verherrlichung einer militärischen Elite, ,einer Handvoll von Soldaten, die eine archaisch gewordene Kampfform mit der damals neuesten Technik aufführten: Das Duell der Kampfflieger am Himmel, zu dem die Soldaten in den Schützengräben hinaufsahen" (WIDDIG 1992:17). Ein Versuch, individuellen Heroismus ideologisch zu retten, war der Mythos vom Jagdflieger als Kampfhelden. ${ }^{6}$ Der Jagdflieger ist ein ganz neuer Kämpfer, ein ,Ritter des Himmels“ in seiner fliegenden Maschine, Objekt der Bewunderung und romantischer Sehnsucht. Zugleich verkörpert er die Tradition von Ehre und Ritterlichkeit. KARL KRAUS (1986:402)

$6 \quad$ Ein anderes Beispiel für das Bild einer nahezu unangefochten triumphierenden Männlichkeit ist der U-Boot-Fahrer als Inkarnation einer geglückten Verbindung von Jugend, Angriffsgeist, Todesbereitschaft und Technik. 
notiert zum fliegenden Helden: „Held ist einer, der gegen viele steht. Diese Position erringt im neuen Krieg am ehesten der Luftbomberwerfer, einer, der sogar über vielen steht.“ Der „Kampf am Himmel“, die Überlegenheit der Vogelperspektive, das Privileg des Überblicks verleihen den Jagdfliegern eine besondere Aura, die - wie MOSSE (1997:155) feststellt - von besonderer Zählebigkeit ist: ,[S] $[$ ogar nach dem Zweiten Weltkrieg bekommen wir zu hören, die Jagdflieger seien wie die Götter und Helden der germanischen Sagen, die den Krieg im Himmel ausgetragen hätten, die modernen Kampfpiloten seien Götter aus Fleisch und Blut." Die himmlischen Kriegshelden, die als eine gelungene Verbindung von Heros und Technik dargestellt wurden, galten als Inbegriff wahrer Männlichkeit, die kühle Professionalität impliziert. Eine bestimmte Physiognomie, ein fester Blick, Beherrschtheit und Anpassung an die neue Dynamik der Kriegsführung gehörten zur Verherrlichung des soldatischen Mannes. 1932 schreibt JÜNGER (1964/6:119) im Essay Der Arbeiter über das Gesicht des Soldaten - und diese Stelle bezeugt die Entwicklung eines neuen Typus des Kriegers:

Es hat in der Skala seiner Ausführungen, wie sie etwa in einer Versammlung oder auf Gruppenbildern zu beobachten ist, an Mannigfaltigkeit und damit an Individualität verloren, während es an Schärfe und Bestimmtheit der ,Einzelprägung“ gewonnen hat. Es ist metallischer geworden, auf seiner Oberfläche gleichsam galvanisiert, der Knochenbau tritt deutlicher hervor, die Züge sind ausgespart und angespannt. Der Blick ist ruhig und fixiert, geschult an der Betrachtung von Gegenständen, die in Zuständen hoher Geschwindigkeit zu erfassen sind.

Im Laufe des Krieges wurde es - auch für solche Künstler und Intellektuelle, die 1914 der Kriegseuphorie verfallen waren - immer spürbarer, ,[...] dass diese selbstgewisse Männlichkeit alles andere als souverän war und außer ihren harten auch ihre weichen Seiten hatte" (SCHILLING 2002:270). Johannes R. Becher verweist in seinem Gedicht Der Krieg auf die Gesichtslosigkeit des Soldaten: Das Gesicht verwächst mit dem Stahlhelm. Kirchner, Grosz und Dix malen verkrüppelte Veteranen und Soldaten als „Zuhälter des Todes“. ${ }^{7}$ Andere bekannte Beispiele der Demontage der heroisierenden Kriegsideologie, die mit der Vision einer starken Männlichkeit einhergeht, sind die Gedichte An Deutschland von Johannes R. Becher, Totentanz von Hugo Ball und Legende vom toten Soldaten von Bertolt Brecht. In ERICH MARIA REMARQUEs (1999:22) deklariert unpolitischem Bestseller Im Westen nichts Neues machen sich die jungen Frontsoldaten darüber lustig, was ihr Lehrer Kantorek in seinem Brief schreibt: „Wir wären die eiserne Jugend.“ Und über die militärische

7 Vgl. das Bild von George Grosz Zuhälter des Todes (1919). 
Ausbildung heißt es an einer Stelle: „Grüßen, Strammstehen, Parademarsch, Gewährpräsentieren, Rechtsum, Linksum, Hackenzusammenschlagen, Schimpfereien und tausend Schikanen: wir hatten uns unsere Aufgabe anders gedacht und fanden, daß wir auf das Heldentum wie Zirkuspferde vorbereitet wurden. Aber wir gewöhnten uns bald daran." (REMARQUE 1999:25) Aus jungen sensiblen und hoffnungsvollen Menschen werden „Menschentiere“, die „zerstören und töten“, um sich zu retten oder zu rächen (83). Sie vertrauen keinem ideologischen Geschwätz, nur dem Zufall, der darüber entscheidet, ob man getroffen wird oder nicht. Sie sind „gefühllose Tote“, die nur zu dreierlei in der Lage sind: „Kartenspielen, fluchen und Krieg führen“ (68) - übrigens ausgesprochen ,männliche' Aktivitäten. Das Beste, was der Krieg hervorgebracht hat, ist männliche Kameradschaft, Freundschaft, Solidarität - die Front hat männliche Gemeinschaften gefördert, was in REMARQUEs (1999:145) Roman deutlich durchschimmert: „,Sie sind mehr als mein Leben, diese Stimmen, sie sind mehr als Mütterlichkeit und Angst, sie sind das Stärkste und Schützendste, was es überhaupt gibt: es sind die Stimmen meiner Kameraden." Die Erfahrung der Kameradschaft bildet eine warme Nische in der Kälte des technisierten Krieges (KÜHNE 1996). Generell muss festgehalten werden, dass Remarque - der „militante Pazifist“ (SCHNEIDER 1998) - mit heroischen Männlichkeitsklischees aufräumt und ein Gegenbild zu Jüngers „Stahlgestalten“ schildert. Der Krieg ist bei Remarque ein Ort des Leidens, der Angst, der Verzweiflung und natürlich des Ausführens sinnloser Befehle, aber nicht der Rehabilitation und Selbstvergewisserung von Männlichkeit. Kühle Professionalität, rasendes Kriegertum einer physisch gestählten Männerhorde - was bei Jünger als „Aufregung des Jägers“ und „übermenschlich“ stilisiert wird, erscheint bei Remarque als inhuman und entwürdigend. Remarques Soldaten stellen sich nüchtern den Tatsachen des Krieges. Deshalb wurde dem Letzteren von rechtskonservativen Kreisen der Weimarer Republik vorgeworfen, dass sein Buch ,[...] einen verhängnisvollen Einfluß auf die junge Generation hatte, daß es das edle Gefühl des Patriotismus, den Sinn für das Heldenhafte tötet, diese seit undenklichen Zeiten höchsten Tugenden der teutonischen Rassen“ (REMARQUE 1998:62).

\section{„Ulanen - bildhübsche Kinder“68}

Während die früheren Jahrhunderte in Polen eine ritterliche Variante des Patriotismus hervorbrachten, entwickelte sich im 19. Jahrhundert der Patriotis-

$8 \quad$ Vgl. das bekannte Kriegslied Hej, hej, ułani, malowane dzieci. 
mus eines unterdrückten Volkes, der sich auf das stets bewahrte Bewusstsein nationaler Zugehörigkeit stützen konnte. Dieses Paradigma bedeutete Konspiration, Verschwörungen, Bereitschaft zum ungleichen Kampf, Niederlagen und Heldentod. Im Angesicht des kommenden Weltkrieges erwachen die Hoffnungen auf Unabhängigkeit, und man beruft sich auf die Tradition der nationalen Erhebungen. Józef Piłsudski verkörpert mit seiner Überzeugung, Polen müsse aus eigener Kraft einen Beitrag zu seiner Wiedererstehung leisten, den Hoffnungsträger schlechthin. Er versammelt eine kleine Freischar ergebener Soldaten um sich (,Kaderkompanie'), überschreitet mit ihnen die Staatsgrenze und will einen eigenen Krieg gegen das Russische Reich führen (er gibt die Konstituierung einer rein fiktiven Nationalregierung in Warschau bekannt). Dieses Ereignis wird als Grundstein für die Bildung der Polnischen Legion betrachtet, deren bedeutende militärische Leistungen zur Konstituierung eines Mythos beitragen, der auf den polnischen Waffenruhm der vergangenen Jahrhunderte rekurriert. Der ,Kommandant' stellt eine Autorität dar und erreicht eine hohe moralische Position, die von der Garde überzeugter Kämpfer für die polnische Unabhängigkeit stets aufrechterhalten wird. Piłsudski steht in der Reihe großer Nationalhelden, Männer der Tat und begabter Führer wie Poniatowski oder Kościuszko, die ihre Soldaten in einen aussichtslosen Kampf führen. Und diese können den Kampf gewinnen mit Hilfe von Phantasie, dem Glauben an die eigenen Möglichkeiten, Verachtung für den Tod und restloser Opferbereitschaft (die Bereitschaft, der ,Mutter" Polen und dem ,Vater" Piłsudski zu folgen). Der legendäre Kommandant erscheint oft als Erlöser der romantische Führer, der in der Lage ist, die schlafenden Ritter zu wecken und auf das Schlachtfeld zu führen. Piłsudski wollte nicht als strenger, seelenloser Vorgesetzter wahrgenommen werden, sondern vielmehr als väterliche Instanz, die die Soldaten betreut. Er soll die Soldaten der Legion, von denen ca. $40 \%$ Akademiker waren, zuweilen als ,sentimentale Intelligenzler“ beschimpft haben, und in der Tat gab es unter ihnen zahlreiche sensible Träumer, die sich in der „Mordsaison 1914-1918“ (KANEHL 1922) in der Kaserne wie zu Hause fühlen sollten. Ein sichtbarer Ausdruck von Piłsudskis ,väterlichem“ Habitus und seiner Solidarität mit den einfachen Soldaten war seine viel gepriesene graue Uniform und der Verzicht auf die Pracht und Aura einer Generalsuniform. Piłsudski war ein Produkt der adeligen Kultur und der romantischen Tradition - ,geschwängert von dichterischen Träumen“ (SCHULZ 1935:425), besessen von der Idee der Auferstehung Polens. Die Konstruktion der polnischen militärischen Männlichkeit im Kontext des Ersten Weltkrieges verbindet sich mit Piłsudski, der Polnischen Legion, seiner Legende und der festen Überzeugung des Kommandanten, dass die Armee der einzige verlässli- 
che Garant der polnischen Unabhängigkeit sein könnte. Deshalb bildet er militärische Untergrundorganisationen (z.B. die Schützenmannschaften), die die männliche Jugend auf den Partisanenkrieg vorbereiten sollen. Die Männlichkeit des Krieges wird nicht - wie in Deutschland, wo man versucht, möglichst viele Männer zu Waffenfähigen auszubilden - universalisiert, sondern sie betrifft vor allem eine kleine ,Elite ' von potentiellen Nationalkämpfern und eine breite Masse von polnischen Rekruten (ca. zwei Millionen Wehrpflichtige), die in die Armeen Russlands, Österreichs und Deutschlands zwangsweise eingegliedert werden, um an den Fronten des Krieges auf eigene Landsleute schießen zu müssen. ${ }^{9}$ Die polnische Literatur der Kriegsära hat weder einen Jünger noch einen Remarque hervorgebracht, was als logische Konsequenz der polnischen Hoffnungen auf das ,Morgenrot der Freiheit' bzw. des ,Traums vom Degen' erscheint. Die pazifistischen Bücher haben eher Seltenheitswert (Wittlin, Zegadłowicz u.a.), obgleich der Mythos von den Polen als einem pazifistischen Volk fortdauert und die Polen seit Jahrhunderten als Liebhaber der Kunst des Fechtens gelten, die jedoch nicht zum nationalen Chauvinismus, Raubrittertum und zu Angriffskriegen fähig sind. Der Erste Weltkrieg war für die Polen ein epochales Ereignis, aber aus anderen Gründen als etwa in Deutschland. In der polnischen Literatur wird er nicht als ein so extrem sinnloses und absurdes Ereignis dargestellt, wie das sonst in der Weltliteratur zu beobachten ist. Der Krieg wird vielmehr sentimentalisiert, nicht etwa als ein mörderischer Kampf oder als, Walzwerk der Front' geschildert, sondern als ,schöne Frau', der die enthusiasmierten, ,bildhübschen' Jungs sofort bedenkenlos und mit großer Freude folgen („Krieglein, Krieglein, was bist du für eine Dame!“ - allerdings: Wer sich in sie verliebt, landet im ,kalten $\mathrm{Grab}^{\text {“10 }}$ ). Im Kontext des Krieges entsteht eine ganze Reihe von rührseligsentimentalen Liedern, die den romantischen Ulanen-Mythos aufbauen, den ,Kommandanten “ und seine ,Fuchsstute“ preisen oder den ,blühenden Rosmarin' und die ,weißen Rosen' besingen. Diese Lieder - ein Beispiel für die besondere Wirksamkeit des postromantischen Gesangs - sind in das

9 Vgl. das berühmte Gedicht Ta, co nie zginęta (,Noch nicht verloren") von Edward Słoński, das über das tragische Schicksal der polnischen Soldaten reflektiert, auf eigene Brüder schießen zu müssen (,,ich - dein Feind und du - mein Feind!“). Der einzige Trost ist die Vision des aus dem Blut der Kämpfer wiedergeborenen Polen, das noch „nicht verloren ist“ (ROMANOWSKI 2002:40).

10 Wojenko, wojenko, cózés ty za pani - das bekannte Kriegslied enthält die Anrufung des Krieges als eine Dame. In der polnischen Sprache sind sowohl das Wort ,wojna' (,Krieg') als auch die Verkleinerungsform ,wojenka' (,Krieglein') Feminina. 
Repertoire des nationalen Liedguts eingegangen und erfreuen sich auch nach 1945 einer nicht nachlassenden Popularität. Die Poesie der Polnischen Legion rekurriert sehr stark auf romantische Muster und glorifiziert die mutigen Kavalleristen - die Blüte der polnischen Männlichkeit, hübsche Jungs, die bereit sind, mit Gewehren Geschütze zu stürmen -, wie das etwa in dem anonymen Lied Sensacja, bateria gra! (,Sensation, die Batterie spielt!') deutlich wird:

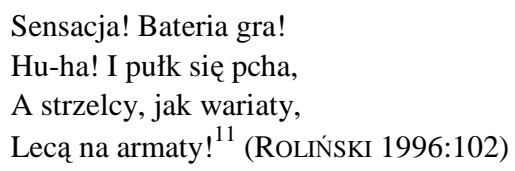

Im 1916 erschienenen Kriegstagebuch von Władysław Orkan Droga Czwartaków (,Auf der Spur des Vierten Infanterieregiments') wird der langwierige Marsch des Vierten Regiments der Polnischen Legion beschrieben. Orkan hat sich im Alter von vierzig Jahren freiwillig gemeldet. Aus seinem Text ergibt sich das Bild einer recht ,weichen', ja sentimentalen Männlichkeit. Die Soldaten bewundern die Landschaft, werden herzlich gegrüßt, marschieren mit Gesang und sind gut gelaunt. Es ist die Rede von soldatischen Herzen und Seelen; besonderen Mut flößt den Soldaten das Regimentsorchester mit dem Marsch des Vierten Regiments ein. Recht bestürzt ist der Erzähler im Angesicht der toten Kameraden: „Wojna - dobrze. Trud wojny - jeszcze. Ale ranni? Zabici?“ (,Der Krieg - gut. Die Mühe des Kampfs - auch gut. Aber die Verwundeten? Die Toten? - ORKAN 2006:106). Eine andere Kriegsreportage von poetischem Charakter ist das Buch von Juliusz Kaden-Bandrowski Pitsudczycy (,Pilsudskis Soldaten'), das in besonderer Weise eine Aura der Einzigartigkeit erzeugt und an der Legende der Legion als einem Dienst, einer Ergebenheit, einem Opfer mitwirkt. Mehrmals ist bei Kaden-Bandrowski die Rede von Piłsudski als einem Mann der Ehre und Gerechtigkeit. Die Befehle des Kommandanten erscheinen als ,gute Ratschläge', die auf der Sicherheit aufbauen, dass man sie aus Überzeugung befolgt:

Trudno powiedzieć, że Piłsudski wydaje rozkazy [...]. Nie tak się to odbywa, a jakoś się dzieje po prostu, poufnie, z dziwną słodyczą. [...] Piłsudski stoi przed kwaterą (śnieżne zbocza gór widnieją dookoła), jedną ręką na płocie wsparty,

11 (,Sensation! Die Batterie spielt! / Hu - Ha! Das Regiment attackiert / Und die Schützen, / nicht bei Sinnen / stürmen die Kanonen.') Die Übersetzungen aus dem Polnischen stammen, sofern nicht anders angegeben, von der Verfasserin. 
drugą głaska lekko po ramieniu wyprężonego, jak struna, dowódcę batalionu. (KADEN-BANDROWKI 1990:12)

Man kann schwer sagen, dass Piłsudski Befehle erteilt [...]. Es funktioniert anders, irgendwie selbstverständlich, es passiert heimlich, mit einer geheimnisvollen Süße. [...] Piłsudski steht vor dem Quartier (rings herum die Bergabhänge im Schnee), mit dem einen Arm auf den Zaun gestützt, streichelt er leicht den Arm des straff gespannten Bataillonsführers.

Mal als ,älterer Bruder' bezeichnet, mal als romantischer ,Denker' und ,Räuber' oder als ,distinguierter Betreuer' bzw. ,Vater', begegnet er seinen Soldaten, seiner ,geliebten Infanterie', mit Härte und Fürsorglichkeit zugleich. Die Kavalleristen - „kwiaty żelazne“ (,eiserne Blumen“ - KADEN-BANDROWSKI 1990:17) - bewegen sich ,furios und schlau' und wissen nicht, was es bedeutet, etwas nicht zu können: „Wola stalowa, wyrastająca z rozpaczy niewolnego piasku [...]“ - (,Der eiserne Wille, aus Verzweiflung des unterdrückten Volks erwachsen [...] ${ }^{\text {‘ }}$ - KADEN-BANDROWSKI 1990:19). KadenBandrowski charakterisiert die Ulanen als mutig und verwegen, in brüderlicher Gemeinschaft miteinander verbunden (das Regiment als zweite Familie), verliebt in Frauen und in Pferde. „Zaś przede wszystkim było to, co się żadną miarą opisać nie da, mianowicie owa gładka, kawalerska uprzejmość, ów zawadiacki, rycerski ton oficerów naszej kawalerii.“"(Und vor allem war es das, was sich in keiner Sprache beschreiben lässt - diese große Freundlichkeit der Kavalleristen, dieser verwegene, ritterliche Ton unserer Offiziere. ' - KADENBANDROWSKI 1990:91). Charakteristisch für die Soldaten der Polnischen Legion war eine besondere Affinität zu Pferden, was in der Tradition der mutigen polnischen Kavallerie steht (ein besonders einprägsames Beispiel ist die berühmte Reiterattacke bei Somosierra). In vielen Gedichten und Liedern werden die polnische Kavallerie und ihre Reiterattacken besungen, besonders der Reiterangriff bei Rokitna (auch als Motiv in der Malerei bekannt, z. B. Wojciech Kossaks Die Reiterattacke bei Rokitna). Die Reiter sind selbstvergessen und todesbereit, sie schwingen ihre Säbel und vertrauen auf ihre eigene Kraft und ihre militärischen Fähigkeiten, auf die Kraft der Idee der Unabhängigkeit Polens, auf das Pferd und auf Gott. Und dann kommen sie in den Himmel, wo sie mit den Engeln tanzen. ${ }^{12}$ Piłsudskis ,Fuchsstute“ ist in die Geschichte

12 Es lassen sich auch Beispiele einer Kritik an einer solchen Determiniertheit und vergeblichen Bravur finden. Schon 1907 machte Żeromski in Sen o szpadzie (,Traum vom Degen') auf die Sinnlosigkeit und Vergeblichkeit der Opfer aufmerksam, und der Held seines Romans Charitas (,Charitas') ist nicht ganz überzeugt vom Sinn des Kampfes, verliert sogar seinen romantischen Enthusiasmus, träumt von der geliebten Frau, leidet und resigniert. 
eingegangen (vgl. z. B. Wacław Kostek-Biernackis Jedzie, jedzie na kasztance - ,Auf der Fuchsstute - oder das Bild von Wojciech Kossak Marschall Józef Pitsudski auf der Fuchsstute). Bolesław Wieniawa-Długoszowski, der in seinen Gedichten und Erinnerungen ein Männlichkeitsbild à la Sienkiewicz kreierte, definiert im Gedicht Moja para (,Mein Paar') die wichtigste Konstellation im Leben des Soldaten der Polnischen Legion: „mein Pferd und meine Geliebte“. ${ }^{13}$ (Zit. nach JODEŁKA-BURZECKI 1989:49) Die polnischen Ulanen sind schön, stolz und erhaben, sie lieben den Säbel, genießen das Leben und haben eine beneidenswerte Phantasie. MAgdalenA MicińsKA (1995:56), die das Bild des polnischen Nationalhelden in der Zeit von 1890 bis 1914 analysiert hat, hält ,,den Imperativ des Kampfes fürs Vaterland, die Todesverachtung und die soldatische Ehre" für die zentralen Werte des ritterlichulanenhaften Universums. Ein besonders stark betontes Element des Ulanenbildes ist die erotische Attraktivität und Anfälligkeit für weibliche Reize. Das populäre Rosmarin-Lied schildert einen Ulanen, der um die Gunst einer Frau wirbt und abgewiesen wird. Er meldet sich zum Militär, bekommt einen Abschiedskuss, nimmt Säbel, Pferd und Schnaps, zieht in den Kampf und fällt. Von WŁADYSŁAW LuDWIK ANCZYC (1918:125) stammt die folgende Charakteristik der polnischen Ulanen:

$$
\begin{aligned}
& \text { śliczne wojsko, jak laleczki, } \\
& \text { zuchowate, śmiałe, } \\
& \text { szare na nich sukmaneczki, } \\
& \text { a wyłogi białe. }{ }^{14}
\end{aligned}
$$

Im Lied Przybyli ułani (,Die Ulanen sind da') werden die prächtigen Jungs von einem Mädchen ins Haus eingeladen, und in Hej, hej ułani (,Hej, Hej, Ulanen“) heißt es: ,ułan chłopak ładny, / ułan chłopak gładki, / kochają go panny, / kochają mężatki“15 (RoMANOWSKI 2002:30). Die Apotheose der Ulanen impliziert notwendigerweise besondere Liebesfähigkeit - sie soll mit den ideologisch erwünschten militärischen Qualitäten einhergehen. Der polnische Ulan, der an die Front geht, nimmt keinesfalls Abschied von , verweiblichenden“ sentimentalen Diskursen der Friedenszeit. Im Gegenteil er sehnt sich stets nach der Mutter, der Geliebten, der Heimat und der Arbeit

13 Das lobende Fazit lautet: „Das Mädchen - still und das Pferd ist gehorsam.“

14 ,Hübsches Militär, einfach wie die Puppen, / mutig und verwegen, / trägt die blauen Kleider, / und weiße Aufschläge.

15 ,Hübsch sind die Ulanen, / glatt sind die Ulanen, / freuen sich die Jungfern / und verheiratete Damen. 
als Bauer oder Angestellter. In KADEN-BANDROWSKIs (1990:106) Pitsudczycy heißt es:

Czasem tylko żołnierz w pożegnaniu rękę za siebie do dziewczyny wyciagną̧, jakby ją chciał razem z jej domem, ciepłem i miasteczkiem, ze sobą wziąć w te pola śnieżne i miejsca nieznane.

Manchmal hat der Abschied nehmende Soldat den Arm ausgestreckt zu der Frau, als wollte er sie samt ihrem Haus, ihrer Wärme, ihrem Städtchen mitnehmen in die verschneiten Felder, in unbekannte Orte.

Zum polnischen Paradigma ,homo militans' gehört ein besonderes Verhältnis zu Frauen: Der Soldat wird zum Abschied von der Geliebten geküsst und von der Mutter gesegnet ${ }^{16}$, er folgt der seltsamen „Dame“, die Krieg heißt, um die ,Mutter' Polen zu befreien und zu der Geliebten zurückzukehren. Das, was dazwischen passiert, wird in der Legionenliteratur als heroischer Kampf einer mutigen Schar von Enthusiasten dargestellt. Obwohl die Materialschlacht kein ritterliches Fechten und Sterben erlaubt, ist dieser Mythos sogar im Zweiten Weltkrieg noch aktuell (vgl. z. B. Żukrowskis Erzählung Lotna - ,Lotna'), und die edle Ritterlichkeit des Sienkiewicz-Typus wird gelegentlich bis heute als eine polnische Spezialität betrachtet (BRANDT 2006). 1942 schrieb JÓZEF WITTLIN (1995:42):

Polski wojownik [...] dziedziczy nadal puściznę swych poprzedników spod Kirchholmu, Samosierry i Racławic. Mimo benzyny, silników i całej skomplikowanej mechaniki, trwa w nim nadal stara romantyczna nuta, choć w zmienionej nieco tonacji. Żadne maszyny, żadne silniki nie zdołają wytracić w polskim wojowniku tej nuty, dyskretnej i smutnej, a tak autentycznej jak cała wiedza fachowa, bez której dziś nie sposób wojować.

Der polnische Kämpfer [...] übernimmt den Nachlass seiner Vorgänger von Kirchholm, Somosierra und Racławice. Trotz Benzin, Motoren und der ganzen komplizierten Mechanik lebt in ihm die alte romantische Note fort, wenn auch in einer leicht abgewandelten Form. Keine Maschinen und Motoren vermögen im polnischen Kämpfer diese diskrete und traurige Note abzutöten, weil sie so authentisch ist wie das gesamte Fachwissen, ohne das heutzutage kein Krieg geführt werden kann.

16 Mutterbilder scheinen sowohl auf symbolischer als auch auf realer Ebene den polnischen Kriegsdarstellungen eingeschrieben zu sein. In den Lebensläufen der Legionäre sind die Mütter ganz besondere Gestalten. In WiTTLiNs (2000:210) Das Salz der Erde gerät der angeblich von einem Soldaten beleidigte Feldwebel in Wut und beschimpft die ganze Kompanie, indem er die Mütter beleidigt: „Die Mütter von mehr als zweihundert Menschen beschimpfen - das bedeutet was! Das gibt Selbstgefühl und bereitet Lust.“ 
WiTTLiNs Roman Sól Ziemi (Das Salz der Erde) ${ }^{17}$, der 1935 erschienen ist, zeigt den Krieg aus der Perspektive eines polnischen Soldaten der österreichisch-ungarischen Armee und entlarvt die Absurdität der Idee, Männer in Uniformen zu stecken und an die Front zu schicken (der monströse Traum, die ganze Menschheit zu uniformieren). Das Gewehr ersetzt den Soldaten die Frau, der Brotsack den Bruder und die Feldflasche das Kind. Die Männer werden zu ,verschwitzten, schäumenden, trunkenen Nummern“ (WITTLIN 2000:30), zu Ensembles von brauchbaren Körperteilen, sie haben - wie Piotr Niewiadomski - „eine dicke Haut, aber ein weiches Herz“ (WITTLIN 2000:51). Ihre Körper werden gewogen und gemessen, um dann von solchen Lehrern wie dem Feldwebel Bachmatiuk umgeformt zu werden. Bachmatiuk formt buchstäblich neue Körper, indem er die verweichlichten Rekruten in die Welt der uniformierten Maskerade einführt. „Für Bachmatiuk existieren keine Menschen mehr, es existieren nur Jahrgänge.“ (WITTLIN 2000:287) Ähnlichen Bildern aggressiver-autoritärer Männlichkeit begegnen wir in Żeromskis Charitas (,Charitas'). Bei Wittlin ,[...] wird der Antagonismus Freund-Feind aufgehoben, jeder ist ein Bruder, der vom höchsten Maß der menschlichen Gebrechlichkeit gezeichnet ist: der Notwendigkeit zu sterben“ (NOWICKI 1999:85). Niewiadomski ist ein Analphabet, ein einfacher Mann, der die ideologischen Begründungen des Krieges weder teilt noch versteht - er ist antiheroisch schlechthin: Der schöne Träumer wird kaserniert und bald an die Front geschickt, wobei er sich wie ein abzuschlachtendes Vieh fühlt. Alles, was passieren wird, ist ihm gleichgültig. Und das einzige Gebiet, auf dem er sich als Sieger fühlen konnte, war Magdas Körper (Piotr „besiegte Magda, er fand keinen Widerstand, aber manche Mühe“ - WiTTLIN 2000:139). Männer wie er, die brutal von ihren Arbeitsplätzen gerissen wurden, könnten nicht diesen „,monströsen Mut“ aufbringen (ZEGADŁOWICZ 1927, zit. nach NowICKI 1999:87), der sie in mordende Maschinen verwandeln würde. Den Wittlin'schen Gestus der Dekonstruktion von militärischer Männlichkeit (der das Schema der Konstruktion bloßlegt), illustriert die folgende Passage:

Ein Soldat besteht aus einer Bluse, einer Hose, einem Mantel, aus Schuhen, einem Gewehr, einem Koppel, zwei Patronentaschen, Bajonett, Tornister, Brotsack, einem Spaten oder einer ,Beilpicke', einem Eßgeschirr, einer Feldflasche, zwei Decken, einer Zeltbahn, aus einer großen Menge Lederriemen - und aus sich selbst. Nun, und einer Kappe. Ohne Kappe ist er fast ein Krüppel, ist er wie eine Lampe ohne Schirm, wie ein Stengel ohne Blüte. (WITTLIN 2000:286)

17 Wittlins Roman wird im Folgenden aus der deutschen Übersetzung zitiert. 
Wittlins Soldatenbilder stehen im krassen Gegensatz zur Apotheose der Ulanen und zum Kampfenthusiasmus, der in der Poesie der Polnischen Legion dominiert (vgl. z.B. die Romantik der Ulanenuniform). Die in Das Salz der Erde geschilderten Rekruten hatten noch keine Feuertaufe: „Sie waren Säuglinge mit grauen, kahlen Köpfen, Babys mit Schnurrbärten; die große Mutter Subordination wird sie an ihren Brüsten säugen." (WITTLIN 2000:302)

\section{Drill oder Erziehung?}

Der Erste Weltkrieg war ein maskulines Ereignis par excellence. Er begann im alten, ,romantischen' Stil, der sich bald in einen neuen, modernen, technisierten verwandelte und Pferde und Lanzen durch Schützengräben und Gewehre ersetzen ließ. Der ,totale' Krieg und die Materialschlacht dekonstruierten alte Muster des abenteuerlichen Kampfes bzw. des individuellen Duells. Die polnische Literatur kreierte einen Heros, der in verschiedenen historischen Kostümen auftrat, der nach dem Schwert greifen sollte, um die Mission der Befreiung Polens durchzuführen. Man kann diesbezüglich von einer „Militarisierung" der literarischen Vorstellungskraft sprechen (kriegerisch-soldatische Metaphorik, historische Symbole, z.B. Schwert, Rüstung, Stahl, zu zerreißende Fesseln usw. - OLSZEWSKA 2004:80), aber nicht von einer allumfassenden Militarisierung der Männlichkeit, die des harten maskulinen Kerns, der für die Maskulinität deutsch-preußischer Prägung typisch war, beraubt zu sein scheint. Der polnische ,homo militans ‘ erwartet den Großen Krieg mit fieberhafter Bereitschaft, seine patriotische Pflicht zu erfüllen und ein Blutopfer darzubringen. „Im Augenblick des Kriegsausbruchs lässt sich ein Kult des Soldatentums beobachten, der auf den polnischen nationalen Charakter sarmatischer Prägung zurückgreift. Er wurde durch die Romantik vertieft und gerechtfertigt und begegnet beispielsweise in Sienkiewiczs Trylogia und Pols Mohort." (OLSZEWSKA 2004:86) Es ist ein wichtiges Element der polnischen patriotischen Erziehung auf der symbolischen Ebene, das auch auf die Sozialisation der Männer Einfluss hatte. Auch in der Armee (in der Polnischen Legion) kann von einem Paradigma der Erziehung die Rede sein, das im krassen Gegensatz zum preußischen Drill steht. ${ }^{18}$ Der Führer erscheint bei Kaden-Bandrowski als guter Vater und Betreuer, bei Jünger als „Übermensch“, der sich die Masse der gemeinen Soldaten unterordnet. In der polnischen Literatur werden Krieg und

18 Zu erwähnen ist hier zum einen das deutsche Männerbund-Syndrom (vgl. BLAZEK 2001:171-293) und zum anderen das Selbstverständnis der deutschen Offiziere als männliche Erzieher der (männlichen) Nation. 
Männlichkeit sentimentalisiert und ,verniedlicht'. Es erscheinen Beschreibungen eines beeindruckend schönen und abenteuerlichen Gefechts, eines „Ulanen-Western“ (JANION 1998:33-39) voller Duelle und Reiterattacken, getragen von begeisterten und opferbereiten Männern aus der Generation der unheilbaren Melancholiker, die nach der Erfüllung der Befreiungsmission zu ihren Müttern und Geliebten zurückkehren wollen. ${ }^{19}$

Auch die deutsche Kultur kennt Beispiele der Sentimentalisierung des Krieges oder Rhapsodien auf junge aufopferungsvolle Helden. Walter Flex zeichnet in seiner unter dem Eindruck des Kriegsausbruchs geschriebenen, in Deutschland sehr populären autobiographischen Erzählung Der Wanderer zwischen beiden Welten. Ein Kriegserlebnis das Porträt seines gefallenen Freundes Ernst Wurche, den er unreflektiert zum Idealbild des aus der Jugendbewegung hervorgegangenen deutschen Kriegsfreiwilligen und vorbildlichen Offiziers stilisiert. Die Schönheit des Soldaten ergibt sich hier nicht aus seinem stahlharten Habitus eines Kriegers:

Trotz und Demut, die Anmut des Jünglings, lagen wie ein Glanz über der Haltung des straffen Körpers, dem schlanken Kraftwuchs der Glieder, dem stolzen Nacken und der eigenwilligen Schönheit von Mund und Kinn. Sein Gehen war federnde, in sich beruhende und lässig bewegte Kraft, jenes Gehen, das ,Schreiten' heißt, ein geruhiges stolzes und in Stunden der Gefahr hochmütiges Schreiten. Der Gang dieses Menschen konnte Spiel sein oder Kampf oder Gottesdienst, je nach der Stunde. Er war Andacht und Freude. (FLEX o. J.:5f.)

Bei aller Sentimentalisierung der Männlichkeit wird aber auch bei Flex - wie HANS RUDOLF WAHL (2003:126) bemerkt - der nationale Diskurs in all seiner Härte und Radikalität reproduziert: Die eigene Nation ,[...] wird so verabsolutiert, daß die Existenz anderer Nationen schlicht nicht mehr wahrgenommen wird." Ein anderes Beispiel ist die Legende vom flämischen Langemarck, auf dessen Feldern Tausende junger Freiwilliger im Kampf gegen eine professionelle britische Armee fielen, die als Zeugnis des Patriotismus und Symbol der Aufopferung im Kampf stilisiert wird (KETELSEN 1985). Generell jedoch gilt für die Konstruktion der deutschen Männlichkeit das Paradigma des Stahlhelden, der - aus sinnentleerter Zivilität erlöst - sich an der Front als ,echter Mann`zu bewähren hat, mit kühler Gelassenheit dem Risiko entgegenblickt

19 Solchen Männerbildern begegnet man nicht nur in der Legionen-Literatur, sondern auch in Romanen wie Odznaka za wierna stużbe (, Auszeichnung für treuen Dienst') und Pokolenie Marka Świdy (,Die Generation von Marek Świda') von Andrzej Strug, Koń na wzgórzu (,Ein Pferd auf dem Hügel') von Eugeniusz Małaczewski oder Zielona kadra (,Grüner Kader') von Jerzy Kossowski. 
und die Gefahr genießt. Diese harte kriegerische, virile Maskulinität geht auf die latent militaristische Disposition der wilhelminischen Gesellschaft zurück und ist Antwort auf die große Krise der hegemonialen Männlichkeit, während die polnische ,weiche' und ,emotionale" Männlichkeitsvariante eine Reaktion auf die lange Zeit der Unterdrückung darstellt. Das deutsche maskuline ,Wir ‘ bedeutet eine militärische Macht und eine Gemeinschaft von Stahlhelden mit dem unterschwelligen Bedürfnis nach Männlichkeitsriten, das polnische ,Wir ${ }^{\star}$ bedeutet die Kraft der Emotionen, die nicht unbedingt einer ,typisch männlichen" Affektkontrolle unterliegen, es bedeutet das Bewusstsein einer großen Tat, einer Mission. Für beide kulturellen Kontexte ist der Drang charakteristisch, einer höheren, überindividuellen Sache zu dienen bzw. die Männlichkeit in den Dienst eines Ideals zu stellen (die Idee der Nation), was einen integralen Bestandteil der europäischen Männlichkeitsdefinition im Kontext des Ersten Weltkrieges ausmacht. Beide kulturellen Kontexte haben Mythen von Kämpfern kreiert, die auf eigene Qualitäten und auf eigene Energie setzen (in Polen der phantasievolle Kavallerist, in Deutschland der professionelle Flieger bzw. U-Boot-Fahrer). Um dem Bedürfnis nach Re-Virilisierung nachzukommen und eine neue männliche Ordnung aufzubauen, nimmt der deutsche Soldat (Jüngers „Prinz der Schützengräben“) Abschied von Frauen und Weiblichkeit. Er betrachtet die Feuertaufe als Liebesnacht, während sich die polnischen Ulanen stets nach Müttern und Geliebten sehnen. Die polnische Konstruktion der militärischen Männlichkeit scheint ,Weiblichkeit' zu integrieren und mit der alten Überzeugung, dass Liebe und Weiblichkeit den Heros schwächen, zu brechen.

Der polnische militärische Diskurs „,von der goldenen Zeit“ (DŁUGOSZ 1917) imaginiert auch nach dem Kriege keinesfalls eine neue männliche Rasse, die ,gestählt‘ aus dem Kampf hervorgeht. ,Körperpanzer" und ,metallische Kälte ‘ sind für den polnischen patriotischen Diskurs eher nicht typisch. Der polnische Soldat ist ein Pilger ins Reich der Freiheit, der seine maskulinen Energien für ein großes Kampf-Event aktiviert. Anders in Deutschland: „Aus dem temporären Kämpfer, der nach dem Krieg - wie in den anderen Nationen - den Soldatenrock wieder auszog, wurde [...] in Deutschland der Krieger zum männlichen Lebensberuf schlechthin.“ (REULECKE 2001:90) Mit einer solchen Konstruktion der soldatischen Männlichkeit haben wir es auf der Ebene der literarisch-künstlerischen Repräsentation zu tun (Jünger, Schauwecker, Beumelburg u. a.). Allerdings bezweifelt der Historiker WolfGANG J. MOMMSEN (2004:145) die tatsächliche Geburt eines neuen, stahlharten Männlichkeitstypus, der im Krieg seine eigentliche Lebenserfüllung gesucht habe. MOMMSEN (2004:151) weist das Klischee des „Frontsoldaten“ als eine ,ideologische 
Fabrikation der Nachkriegsjahre" zurück. Sowohl in der deutschen als auch in der polnischen Literatur und Kunst finden sich Beispiele einer desillusionierenden Darstellung des Krieges und der Dekonstruktion der jeweils dominierenden Männlichkeit. Die Poesie der Polnischen Legion meidet sorgfältig die tägliche Mühe und Hässlichkeit des Soldatenlebens und den soldatischen Opferstatus. Aber auch in ihr werden mit der Zeit entheroisierende Akzente bemerkbar, die in den später entstehenden pazifistischen Texten eines Wittlin, Strug oder Zegadłowicz überwiegen. In Deutschland ließen auch pazifistisch gesinnte Schriftsteller ihre Protagonisten zu vorbildlichen Soldaten werden, sie beschworen männliche Kriegskameradschaft und stilisierten soldatische Tugenden (z.B. Erich-Maria Remarque). Ludwig Renn, ein erklärter Kriegsgegner, lässt einen der Protagonisten des Romans Krieg (1929) zu einem vorbildlichen Soldaten mit allen entsprechenden körperlichen und geistigen maskulinen Eigenschaften werden (mutig, stolz, ausdauernd, vielleicht kein wahrer Patriot, aber ein richtiger Mann). Renn zeigt, verdreckte Helden' im unendlichen Einerlei von Märschen, Granatangriffen, Patrouillen, Hunger, Sterben und Verwundetwerden. Es gilt unter diesen widrigen Bedingungen, stoisch seinen Mann zu stehen und die soldatische Pflicht zu erfüllen. Dagegen wird in Leonhard Franks Der Mensch ist gut (1918) nicht nur der Krieg abgelehnt, sondern auch die Männlichkeit in Frage gestellt, die ihm den Boden bereitet hatte. Die sozialistische Männlichkeitsvorstellung bildet einen Gegenpol zur Maskulinität des gewalttätigen Soldatentypus. In den Werken der expressionistischen Dichter und Maler zeigt der Krieger oft seine weichen Seiten, und es kommt zur Entmännlichung der Männer, die Helden werden wollten. Die Männer erscheinen als „Geister der Helden“ (TRAKL 1987:167), verstümmelte Opfer, verletzlich, zerbrechlich - eine anonyme, entpersönlichte Masse. Eben dies ist auch in der polnischen Literatur der Fall, etwa bei Nałkowska oder Nowakowski. Die deutsche und polnische Männlichkeit des Krieges unterscheiden sich im Grad des Maskulinismus, in der Einstellung zu Krieg und Militarismus, zu Frau und Weiblichkeit, zu Staat und Nation. Die Problematik kompliziert sich noch weiter, wenn man die Frage nach hegemonialen Mustern stellt, die sich in historischen und lokalen Kontexten konstituieren. Es scheint sinnvoll zu sein, nicht nur von verschiedenen Männlichkeiten, sondern auch von verschiedenen militärischen Männlichkeiten zu sprechen. Der polnische Männlichkeitsdiskurs scheint stellenweise das zu dekonstruieren, was in Deutschland als harte Ästhetik der Maskulinität des Soldatentypus propagiert wird. Die Einführung der Allgemeinen Wehrpflicht führte nämlich nicht in ganz Mitteleuropa zu einer generellen Militarisierung der hegemonialen Männlichkeit (SCHMALE 2003:195-203), auch wenn überall die Armee als ,Schule 
der Männlichkeit‘ galt und erst die militärische Erfahrung einen ,ganzen Mann' konstituierte. CHRISTA HÄMMERLE (2005) hat das weitgehend in der Forschung angenommene stark verallgemeinernde Modell der hegemonialen militärischen Männlichkeit am Beispiel der ethnisch-kulturell differenzierten Habsburgermonarchie und ihrer ,multi- oder supranationalen' Armee, in der ja auch die Polen gedient hatten, weitgehend relativiert. Es gibt womöglich guten Grund zu vermuten, dass man es im Umfeld des Ersten Weltkrieges mit mehreren hegemonialen Männlichkeiten zu tun hat. Die einzelnen (nationalen) Männlichkeitskonzepte bzw. Konzepte der militärischen Männlichkeit haben eine eingeschränkte Reichweite und die sie (re)produzierenden Diskurse und Praxen sind in lokalen Männlichkeitstraditionen und historischen Kontexten verwurzelt. Drill, Erziehung und/oder eigenwilliger Kampfelan - am Beispiel dieser Alternative kann die Frage nach den nationalen Unterschieden in den Konzepten der militärischen Männlichkeit diskutiert werden.

\section{Literatur}

ANCZYC, WŁADYSŁAw LudwiK (1918): Utan [Der Ulan]. In: Życie i pisma [Leben und Schriften]. Bearbeitet von M. Szyjkowski. Bd. 2. Kraków, 125.

BlazeK, Helmut (2001): Männerbünde. Eine Geschichte von Faszination und Macht. Berlin.

BRANDT, MARION (2006): Motyw polskiego utana / polskiej kawalerii w niemieckiej literaturze lat osiemdziesiatych [Das Motiv des polnischen Ulanen und der polnischen Kavallerie in der deutschen Literatur der 1980er Jahre]. In: MOROZ, GRZEGORZ / Ossowski, MirosŁaw / SZTACHELSKA, JolanTA (eds.): Tożsamość i odrębność w Zjednoczonej Europie. Obrazy krajów i stereotypy narodowe $w$ literaturze anglo- $i$ niemieckojęzycznej. Olecko, 175-191.

CONNELl, RoBert W. (1999): Der gemachte Mann. Konstruktion und Krise von Männlichkeiten. Übersetzt von Christian Stahl. Opladen.

DEHMEL, RICHARD (1914): Lied an alle. In: Frankfurter Zeitung, 4.08.1914.

DŁugosz, StanisŁaw (1917): Przed złotym czasem. [Vor der goldenen Zeit]. Kraków.

FleX, Walter (o.J.): Der Wanderer zwischen beiden Welten. Ein Kriegserlebnis. München.

FREVERT, UTE (1991): Ehrenmänner. Das Duell in der bürgerlichen Gesellschaft. München.

HÄMmerle, Christa (2005): Zur Relevanz des Connell'schen Konzepts hegemonialer Männlichkeit für „Militär und Männlichkeit/en in der Habsburgermonarchie (18681914/18) “. In: DingeS, MARTIN (ed.): Männer - Macht - Körper. Hegemoniale Männlichkeiten vom Mittelalter bis heute. Frankfurt (M.)/New York, 103-121. 
Monika Szczepaniak

Hagemann, Karen (1996): „Heran, heran, zu Sieg oder Tod!“ Entwürfe patriotischwehrhafter Männlichkeit in der Zeit der Befreiungskriege. In: KÜHNE, THOMAS (ed.): Männergeschichte - Geschlechtergeschichte. Männlichkeit im Wandel der Moderne. Frankfurt (M.)/ New York, 51-68.

HANISCH, ERNST (2005): Männlichkeiten. Eine andere Geschichte des 20. Jahrhunderts. Wien/Köln/Weimar.

JAnion, Maria (1991): Polski korowód. [Der polnische Reigen]. In: TAZBIR, JANuSZ (ed.): Mity i stereotypy w dziejach Polski. Warszawa, 185-242.

- (1998): Wojna i forma. [Krieg und Form]. In: JANION, MARIA: Płacz generata. Eseje o wojnie. Warszawa, 23-126.

JodeŁKa-Burzecki, Tomasz (ed.) (1989): Poezja legionowa. Antologia. [Poesie der Polnischen Legion. Eine Anthologie]. Warszawa.

JÜNGER, ERNST (1922): Der Kampf als inneres Erlebnis. Berlin.

- (1964): Werke. Bd. 6: Essays II. Der Arbeiter. Stuttgart.

Kaden-Bandrowski, Juliusz (1990): Pitsudczycy. [Piłsudskis Soldaten]. Białystok.

KANEHL, OsKar (1922): Die Schande. Gedichte eines dienstpflichtigen Soldaten aus der Mordsaison 1914-1918. Berlin.

Ketelsen, Uwe-K. (1985): „Die Jugend von Langemarck. “ Ein poetisch-politisches Motiv der Zwischenkriegszeit. “ In: KoEBner, ThOMAs (ed.): „Mit uns zieht die neue Zeit. “ Der Mythos Jugend. Frankfurt (M.), 68-96.

Kraus, KaRL (1918): Das technoromantische Abenteuer. In: Die Fackel 474-483:4145 .

- (1986): Aphorismen. Sprüche und Widersprüche. Pro domo et mundo. Nachts. Frankfurt (M.).

Krockow, Christian Graf VON (1995): Von deutschen Mythen. Stuttgart.

KÜHNE, ThOMAS (1996): „...aus diesem Kriege werden nicht nur harte Männer heimkehren." Kriegskameradschaft und Männlichkeit im 20. Jahrhundert. In: KüHNE, ThOмAs (ed.): Männergeschichte - Geschlechtergeschichte. Männlichkeit im Wandel der Moderne. Frankfurt (M.), 174-192.

Lethen, Helmut (1990): Kältemaschinen der Intelligenz. Attitüden der Sachlichkeit. In: WICHNER, ERNEST / WIESNER, HERBERT (eds.): Industriegebiet der Intelligenz. Literatur im neuen Berliner Westen der 20er und 30er Jahre. Ausstellungsbuch. Berlin, 119-153.

MicińSKa, Magdalena (1995): Między Królem Duchem a mieszczaninem. Obraz bohatera narodowego w piśmiennictwie polskim przełomu XIX i XX wieku (1890-1914). [Vom König Geist zum Bürger. Das Bild des Nationalhelden in der polnischen Literatur der Jahrhundertwende (1890-1914)]. Wrocław.

Mommsen, Wolfgang J. (2004): Der Erste Weltkrieg. Anfang vom Ende des bürgerlichen Zeitalters. Frankfurt (M.). 
Mosse, George L. (1997): Das Bild des Mannes. Zur Konstruktion der modernen Männlichkeit. Frankfurt (M.).

Nowicki, Bogdan (1999): Ekspresjonizm polski wobec Pierwszej Wojny Światowej. Kasprowicz - Przybyszewski - Zegadłowicz - Wittlin. [Der polnische Expressionismus und der Erste Weltkrieg. Kasprowicz - Przybyszewski - Zegadłowicz - Wittlin]. In: Łoch, Eugenia / STĘPNIK, KRZYSzTOF (eds.): Pierwsza wojna światowa w literaturze polskiej i obcej. Wybrane zagadnienia. Lublin, 81-92.

OlszewsKa, MaRia Jolanta (2004): Człowiek w świecie Wielkiej Wojny. Literatura polska z lat 1914-1919 wobec I wojny światowej. Wybrane zagadnienia. [Der Mensch im Universum des Großen Krieges. Polnische Literatur der Zeit 1914-1919 und der Erste Weltkrieg. Ausgewählte Probleme]. Warszawa.

ORKAN, WŁADYSŁaw (2006): Droga Czwartaków od Ostrowca na Litwę. [Auf der Spur des Vierten Infanterieregiments von Ostrowiec nach Litauen]. Kraków.

RadKau, JoAchim (1998): Das Zeitalter der Nervosität. Deutschland zwischen Bismarck und Hitler. München/Wien.

Remarque, ERICH MARIA (1998): Haben meine Bücher eine Tendenz? In: SCHNEIDER, Thomas F. (ed.): Erich Maria Remarque. Ein militanter Pazifist. Texte und Interviews 1929-1966. Köln, 62-65.

- ( 1929 / 1999): Im Westen nichts Neues. Roman. Mit einem Nachwort von Tilman Westphalen. Köln.

REULECKE, JÜRGEN (2001): „Ich möchte einer werden so wie die... “. Männerbünde im 20. Jahrhundert. Frankfurt (M.).

RoliŃSKI, AdAM (ed.) (1996): A gdy na wojenke szli ojczyźnie stużyć... Pieśni i piosenki żotnierskie z lat 1914-1918. Antologia. [Als sie in den Krieg gingen, um dem Vaterland zu dienen... Polnische Soldatenlieder der Zeit 1914-1918. Eine Anthologie]. Kraków.

RomAnowski, ANDRZEJ (ed.) (2002): My, Pierwsza Brygada... Mała antologia poezji $i$ pieśni I Wojny Światowej. [Wir, die Erste Brigade... Eine Kleine Anthologie von Gedichten und Liedern aus dem Ersten Weltkrieg]. Kraków.

SCHILling, RENÉ (2002): „Kriegshelden“. Deutungsmuster heroischer Männlichkeit in Deutschland 1913-1945. Paderborn.

Schmale, Wolfgang (2003): Geschichte der Männlichkeit in Europa (1450-2000). Wien/Köln/Weimar.

Schulz, Bruno (1935): Powstaja legendy. [Legenden entstehen]. In: Tygodnik ilustrowany 22:425.

TheEl, ROBert (1993): „Die Maschine hat den Helden getötet. “ Beobachtungen zu direkten und indirekten Verwendungen des Mentalitätsbegriffs in fiktionalen und essayistischen Texten vor und während des 1. Weltkrieges im Hinblick auf den Heroismusbegriff (Nowak, Soyka, Kraus, Unruh, Marinetti, Rilke). In: Krieg und Literatur / War and literature 9:97-118.

TheWeleit, Klaus (1980): Männerphantasien. Bd. 2. Reinbek bei Hamburg. 
Monika Szczepaniak

Trakl, Georg (1915): Grodek. In: Trakl, Georg: Dichtung und Briefe. Historischkritische Ausgabe. Hrsg. von Walter Killy und Georg Szklenar. Bd. 1. Salzburg, 167.

Wahl, Hans Rudolf (2003): „Der Sturm geht über die Gräber in Polen. “ Anmerkungen zu Walter Flex. In: Studia Germanica Gedanensia 11:113-129.

WIDDIG, BERND (1992): Männerbünde und Massen. Zur Krise männlicher Identität in der Literatur der Moderne. Opladen.

WitTLin, Józef (1995): Wojna i stowo. [Krieg und Wort]. In: Eseje rozproszone. Hrsg. und bearb. von Paweł Kędziela. Warszawa, 41-42.

- (2000): Das Salz der Erde. Roman. Aus dem Polnischen von Izydor Berman. Frankfurt (M.).

Worringer, Wilhelm (1914/15): Ohne Titel. In: Zeit-Echo. Ein Kriegstagebuch für Künstler 2:22-29.

ZEGADŁOWICZ, EMIL (1927): Dziewanny. [Königskerzen]. Warszawa. 